\title{
An Application of Spearman's Rho in Blind Deconvolution of Seismic Data
}

\author{
Rongrong Wang ${ }^{1, a^{*}}$, Fei Xu ${ }^{1, b}$ and Xiaobo Zhou ${ }^{1, c}$ \\ ${ }^{1}$ Electronic information Engineering Department, Xijing University, Shaanxi Xi'an, China

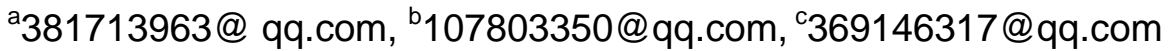 \\ * The corresponding author
}

Keywords: Spearman's rho; Seismic wavelet; Blind deonvolution; Mutual information; Inverse filter

\begin{abstract}
The paper proposes a novel seismic blind deconvolution approach based on the Spearman's rho in the case of band-limited seismic data with a low dominant frequency and short data records. The spearman's rho is a measure of the dependence between two continuous random variables without the influence of the marginal distributions, by which a new criterion for blind deconvolution is constructed. The optimization program for new criterion of blind deconvolution is performed by applying Neidell's wavelet model to the inverse filter. The simulation test and actual seismic data processing results show that this method can effectively expand the frequency band of seismic records, and enhance the resolution of seismic date.
\end{abstract}

\section{Spearman's Rho 在地震盲反褶积的应用}

\author{
王荣荣 ${ }^{1, a}$, 徐飞 ${ }^{1, b}$, 周晓波 ${ }^{1, c}$ \\ 1. 西京学院电子信息工程系, 中国陕西西安 710123 \\ 3381713963@qq.com, ${ }^{\mathrm{b}} 107803350 @ q q . c o m,{ }^{\mathrm{c}} 369146317 @ q q . c o m$
}

摘要: 针对带限短数据集的地震信号处理问题, 提出了一种基于 Spearman's rho 的盲反褶积 算法。依据 Spearman's rho 可以度量无边缘分布影响的两个连续随机变量之间相关性原理, 构造了新的盲反褶积度量标准。该盲反褶积优化准则中逆滤波器采用 Neidell 小波模型。仿真 实验及对实际数据的处理都验证了该算法可以有效扩展地震记录的频带, 提高地震资料的分 辨率。

关键词: Spearman’s rho；地震子波；盲反褶积；互信息；逆滤波器

\section{1. 引言}

在地震数据处理中，观测到的地震数据被表示成地震子波与地层反射系数的褶积，反褶积处 理是地震数据处理的一个重要环节 $[1,2]$, 其主要目地是试图恢复被地震子波滤波后的反射系 数序列, 也就是提高地震勘探的纵向分辨率。在褶积模型中地震子波未知, 这属于盲反褶积 问题。统计性反褶积是常用的一种盲反褶积方法, 它是充分利用了反射系数及地震记录的统 计信息来进行反褶积处理的。长期以来, 人们对这类算法做了大量的研究, 提出了不同种类、 基于各种信息准则的反褶积算法 [3-6]。

如果褶积算子波在单位圆上有零点, 盲反褶积就是一个不适定问题, 适用于逆滤波器的准则 之一是反褶积的输出信号之间的独立性最大。互信息是一个衡量随机过程独立性的准则, 不 受边缘分布影响。如果变量之间的互信息率为零，变量之间是相互独立的。Larue 提出了一种 基于互信息率最小化的单输入单输出盲反褶积方法 [7]。对于有噪声的带限地震信号, Baan 和 Pham [8]提出了一种修改互信息率反褶积方法。基于极小化互信息率的反褶积算法首先需 要估计输出信号的概率密度函数, 进而得到对输出信号熵的梯度的估计。如果没有待估计信 号概率密度的先验知识, 需要的计算量与数据长度的平方成正比。地震信号是典型的非平稳 
信号, 仅仅在一定的时窗内可以近似认为平稳, 所以在一个地震道中, 视为平稳的数据集长 度可能较短, 对此数据概率密度估计的精度会下降, 这使得该算法不适用于时变地震信号的 反褶积。

在现代统计理论中, 互信息只是度量数据之间独立性的方法之一。有很多种度量都可以表示 随机变量的相互关系, 它们的特点是在单调变换下可以保持度量不变, 在处理盲反褶积问题

上, Spearman's rho $\left(\rho_{X, Y}\right)[9]$ 是一个有用的工具。本文主要目的是构建一个基于 Spearman's rho 的地震反褶积准则, 并提出一种新的盲反褶积算法。该方法可以处理小样本的数据, 意味 着它具有处理非平稳的数据的能力。

\section{2. 基本理论}

\subsection{Spearman's rho}

如果两个随机变量 $X, Y$ 具有分布函数 $F, G, x, y$ 分来自 $X, Y$, 使 $u=F(x), v=G y)$, Spearman's rho $\left(\rho_{X, Y}\right)$ 的表达式可以写成以下形式:

$\rho_{X, Y}=\operatorname{cov}(U V)$

对于平稳的地震信号 $\left\{x_{n}\right\}$, 地震道的每一个样本具有相同的分布函数。经验分布函数可以应 用于计算相应样本 $x_{n}$ 的 $\mathrm{u}_{n}$

$u_{n}=\frac{1}{N} \sum_{i=0}^{N-1} 1\left\{x_{i} \leq x_{n}\right\}$

其中 $1\{A\}$ 是集合 $\mathrm{A}$ 的指示函数, $\mathrm{N}$ 表示数据的长度。

2.2. 逆滤波器

根据 Baan and Pham 的研究, 知道如果子波是已知的, 维纳滤波器是最优的逆滤波器, 其在 频域中，其表达式可以写 W 为:

$$
A_{o p t}(\omega)=\frac{\bar{W}(\omega)}{|W(\omega)|^{2}+\varepsilon^{2}}
$$

其中 $W$ 为子波的傅里叶变换, $\varepsilon^{2}$ 为正则化因子, $\bar{W}$ 代表 $W$ 的复共轭。

实际中地震子波未知, 所以盲反褶积过程是一个估计地震子波的过程。采用 Neidell[9] 的小 波模型来构建逆滤波器。Neidell[10] 基于实际对地震子波的观测, 提出一种表达式极为简洁 的子波模型, 该子波族能够很好地吻合实际的地震子波, 其 $\mathrm{Z}$ 变换形式为:

$W(Z)=(1-Z)^{n}(1+Z)^{m}$

其中 $m$ 和 $n$ 的关系为

$\cos \omega_{p}=\frac{m-n}{m+n}$

$\omega_{p}$ 为子波振幅谱的峰值频率。上式表明, 如果知道了地震信号的峰值频率, 要优化确定的参 数只有一个。在实践中, 通常不知道这个峰值频率。因此, 有以下值作为它的替换:

$\hat{\omega}_{p}=\underset{\omega}{\arg \max }\left|F T\left(x_{n}\right)\right|$

其中, FT 表示傅里叶变换。 


\section{3. 新的反褶积算法}

根据上面的的分析结果，这里提出一种基于新的频域准则的盲反褶积算法。

$$
J(A)=\operatorname{cov}\left(u_{n} u_{n+l}\right)
$$

$I$ 表示时间延迟。确定了 $\omega_{p}$ 后， $J(A)$ 是 $\mathrm{n} 、 \mathrm{~m}$ 的函数，因此，有

$$
n_{\text {opt }}=\underset{n}{\arg \min } J(A)
$$

$$
A_{o p t}(\omega)=\frac{\bar{W}_{N}\left(n_{o p t}, \omega\right)}{\mid W\left(n_{o p t}, \omega\right)^{2}+\varepsilon^{2}}
$$

因此，提出一种新的反褶积算法步骤如下:

(1) 估计地震数据 $\left\{x_{n}\right\}$ 的 $\omega_{\mathrm{p}}$ 。

(2) 计算不同的 $n$ 值对应的逆滤波器的输出 $\left\{\boldsymbol{u}_{n}^{n}\right\}$, 其中上标代表逆滤波器的参数, 下标表 示时间序列, $\mathrm{u}_{\mathrm{n}}$ 由（2）给出, 逆滤波器由（3）和（4) 给出。

(3) 对于一个合适的 1 , 计算代价函数 $J$ 对应的逆滤波器参数 $n$ 。

(4) 搜索最大代价函数获得最优逆滤波器。

\section{4. 算例}

\section{1. 仿真算例}

通过高斯反射系数与 $40 \mathrm{~Hz}$ 的雷克子波卷积来仿真地震记录, 抽样时间间隔 $1 \mathrm{~ms}$ 。为检验该算 法对短数据集的适应能力，选择合成记录的长度约为 $200 \mathrm{~ms}$ ，反褶积结果如图 1 。

如图 1 所示，估计子波几乎与真实子波重合，小波估计的非常精确，原始的频谱范围为 $0 \mathrm{~Hz}$ 到 $100 \mathrm{~Hz}$ ，反褶积后频谱范围为 $0 \mathrm{~Hz}$ 到 $200 \mathrm{~Hz}$ 。地震记录的频谱明显被有效拓宽。
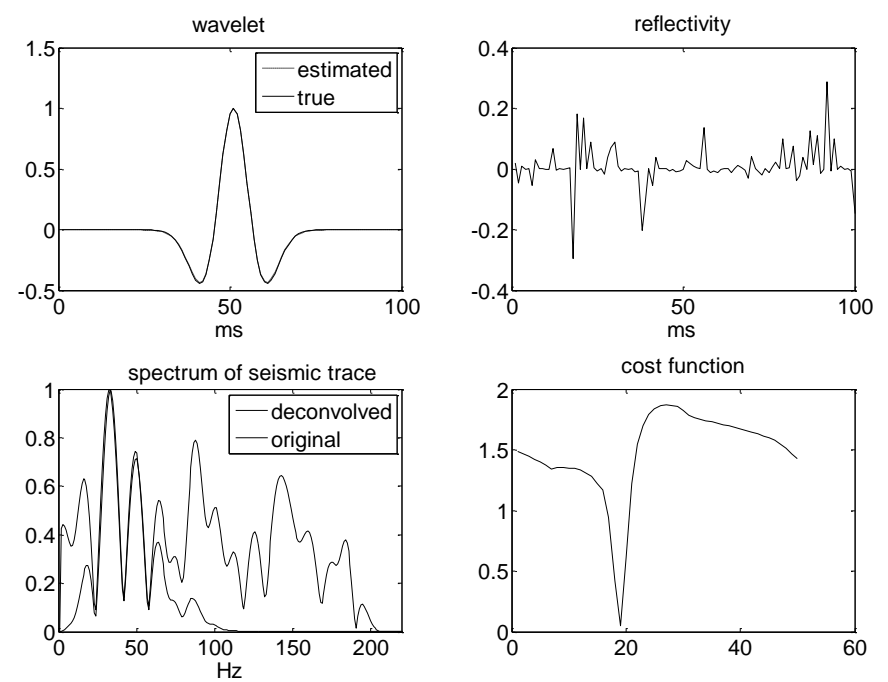

图 1 无噪下的反褶积处理结果

为了测试该算法的抗噪声的能力, 这里采用合成噪声信号, 信噪比为 $15 \mathrm{~dB}$, 结果如图 2 所示。 图 2 为信噪比为 $15 \mathrm{~dB}$ 时的处理结果, 由于噪声的存在, 对子波的估计精度下降, 但吻合的 也很好，地震记录的频谱也得到了拓宽，说明了该算法的抗噪性。 

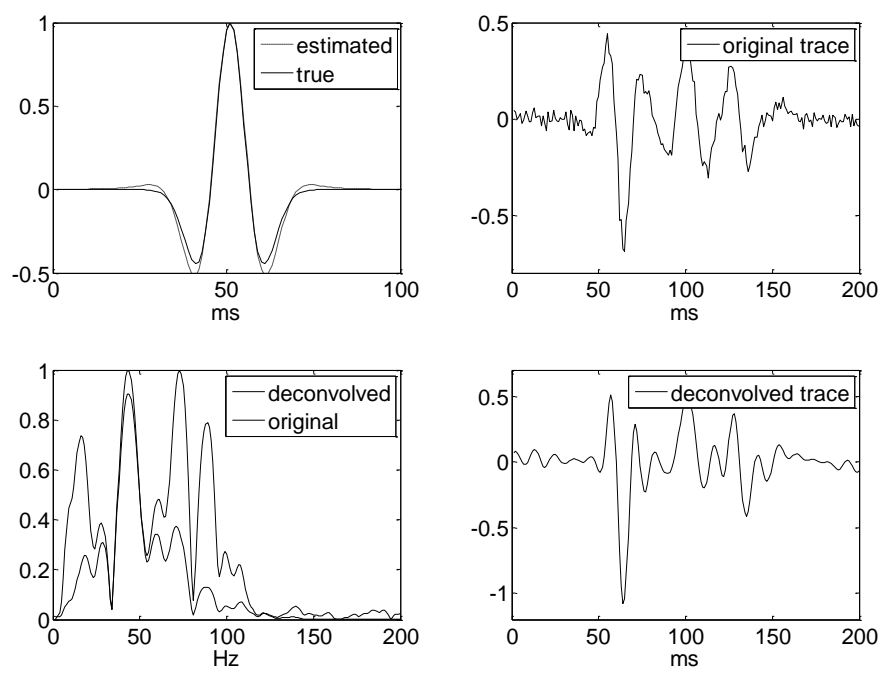

图 2 有噪声信号的反褶积处理结果

\section{2. 单道数据处理}

用该方法处理一个来自胜利油田的单道实际地震数据, 原始频谱的范围 $0 \mathrm{~Hz}$ 至 $120 \mathrm{~Hz}$, 而反 褶积谱的范围 $0 \mathrm{~Hz}$ 到 $180 \mathrm{~Hz}$ 。在与原始记录的频谱相比, 反褶积的频谱明显变宽, 见图 3 。

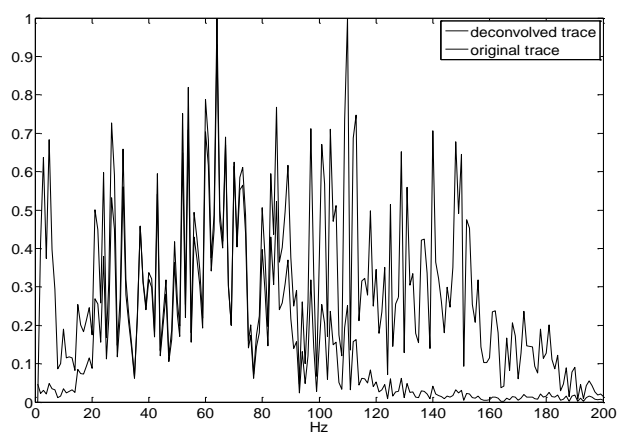

\section{图 3 原始地震道的振幅谱和反褶积结果对比}

\section{3 . 叠加剖面数据处理}

这里处理一个实际剖面地震数据来检验本文方法的性能, 该剖面信号有 50 道数据, 采样间隔 为 $1 \mathrm{~ms}$, 图 4 显示了原始数据和使用本文方法反襀积后的结果, 反褶积后的图像分辨率明显 改善。
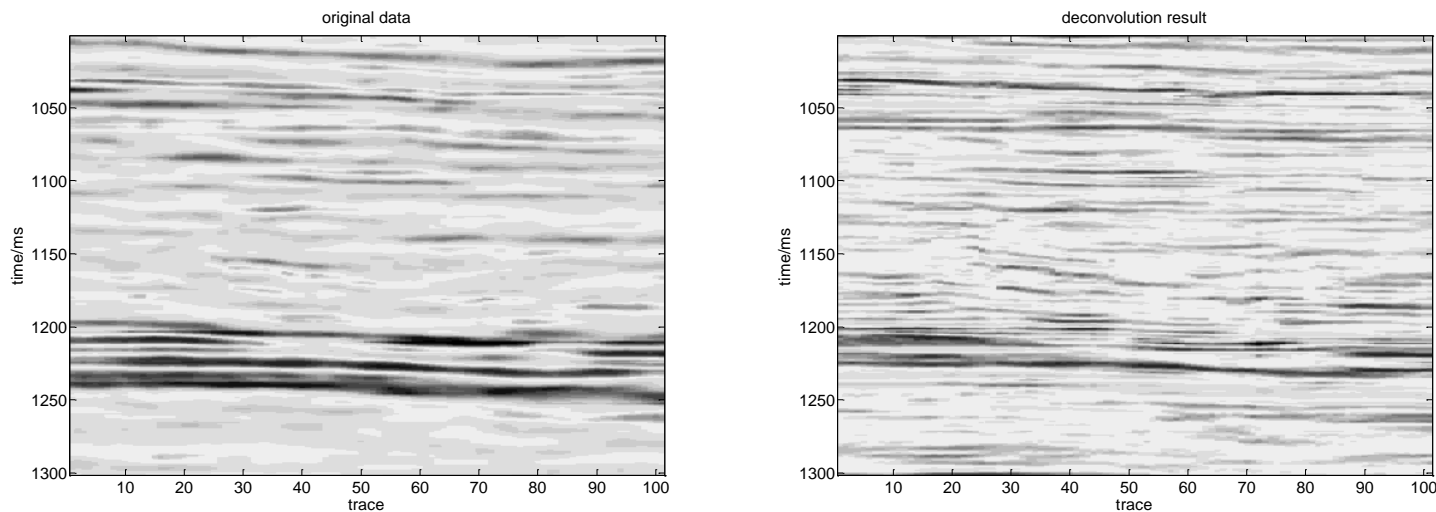

图 4 原始数据和反褶积处理后对比 


\section{5. 结论}

在本文中, 提出了基于两个连续随机变量的 Spearman' s rho 度量的盲反褶积方法, 构造了 新的盲反褶积准则。通过仿真信号及两个实际例子的验证的, 显示了将该方法用于对非平稳 地震信号处理中可以取得很好的效果。

\section{6. 致谢}

感谢陕西省教育厅专项科研计划项目 (2013JK1121) 对本研究的支持。

\section{参考文献}

[1] 刘喜武, 刘洪. 地震盲反褶积综述[J]. 地球物理学进展, 2003,18(2)：203-209.

[2] 左博新, 胡祥云, 韩波. 基于褶积模型的地球物理反演模型增强 [ $\mathrm{J}]$. 地球物理学报, 2012,55(12): 4058-4068.

[3] 高静怀, 张明, 张兵. 一种改进的基于互信息率的盲反褶积方法 [J]. 地球物理学进展, 2011, 26(2): 540-548.

[4] 蔡连芳, 田学民. 基于峭度最大化的地震盲反褶积方法 [J]. 石油物探, 2012,51(1): 30-36.

[5] Zhang B, Gao J H. Blind Deconvolution of Seismic Data Using f-Divergences[J]. Entropy, Vol.13(2011), No.9, pp.1730-1745.

[6] Wiggins R.A. Minimum entroy deconviution [J]. Geoexploration,Vol.16(1978),pp.21-35.

[7] Larue A, Mars. J.I and Jutten C. Frequency-Domain Blind Deconvolution Based on Mutual Information Rate[J]. IEEE transactions on signal processing,Vol.54(2006), No.5,pp.1771-1781.

[8] Baan M V, Pham D T. Robust wavelet estimation and blind deconvolution of noisy surface seismics[J]. Geophysics, Vol.73 (2008), No.5, pp.V37-V46.

[9] 王沁, 王璐, 袁代林, 基于Spearman的rho的Copula参数模型的选择 $[\mathrm{J}]$. 数学的实践与认 识, 2011,41(15): 145-150.

[10]Neidell N S. Could the processed seismic wavelet be simpler than we think?[J]. Geophysics, Vol.56(1991), No.5, pp.681-690.

\section{Acknowledgement}

Scientific Research Program Funded by Shaanxi Provincial Education Department (Program No. 2013JK 1121).

\section{References}

[1] Liu Xi-wu, Liu Hong. Survey on seismic blind deconvolution[J]. Process in geophysics, Vol.18(2003), No.2, p.203-209 (in Chinese)

[2] Zuo Bo-xin, Hu Xiang-yun, Han Bo. The geophysical model enhancement based on the convolution model [J]. Chinese Journal of Geophysics, Vol.55 (2012), No.12, p.4058-4068 (in Chinese)

[3] Gao Jing-huai, Zhang Ming, Zhang Bing. An improved blind deconVolution based On mutual information rate [J]. Process in geophysics, Vol.26 (2011), No.2, p.540-548 (in Chinese)

[4] Cai Lian-fang, Tian Xue-min. A seismic blind deconvolution method based on kurtosis maximization criterion[J]. Geophysical Prospecting for Petroleum, Vol.51(2012),No.1, p.30-36 (in Chinese) 
[5] Zhang B, Gao J H. Blind Deconvolution of Seismic Data Using f-Divergences [J]. Entropy, Vol.13(2011), No.9, pp.1730-1745.

[6] Wiggins R.A. Minimum entroy deconviution[J]. Geoexploration,Vol.16(1978), pp.21-35.

[7] Larue A, Mars. J.I and Jutten C. Frequency-Domain Blind Deconvolution Based on Mutual Information Rate [J]. IEEE transactions on signal processing, Vol.54 (2006), No.5, pp.1771-1781.

[8] Baan M V, Pham D T. Robust wavelet estimation and blind deconvolution of noisy surface seismics [J]. Geophysics, Vol.73 (2008), No.5, pp.V37-V46.

[9] Wang Qin, Wang Lu, Yuan Dai-lin. Choice of the Copula Parameter Model Based on Spearman's Rho [J]. Journal of Mathematics in Practice and Theory,Vol.41(2011), No.15, p.145-150 (in Chinese)

[10] Neidell N S. Could the processed seismic wavelet be simpler than we think? [J]. Geophysics, Vol.56 (1991), No.5, pp.681-690.

作者简介: 王荣荣 $(1971-)$, 女, 陕西, 讲师, 主要从事信号监测、信号及信息处理方法 及应用灯方面的研究工作, E-mai1：381713963@qq. com。 